\title{
The Effect of Socio-Economic Status and Gender on Adolescent Anger in Chittagong
}

\author{
${ }^{1}$ Abdur Rahman, ${ }^{2}$ Arunavo Bairagi, ${ }^{3}$ Biplob kumar Dey \\ ${ }^{1,2,3}$ Department of Psychology, University of Chittagong, Bangladesh.
}

\begin{abstract}
The present study attempted an empirical investigation to explore the effect of socio- economic status and gender on adolescent anger at Chittagong in Bangladesh. A total 120 respondents constituted the sample of the study. Among them $60(50 \%)$ adolescents were male and $60(50 \%)$ were female. The respondents came from different socio-economic status. 40 (33.3\%) adolescents were from higher class, 40 (33.3\%) from middle class and the rest of 40 (33.3\%) were from lower class. An adapted Bangla version of "Adolescent Anger Rating Scale" (AARS) was used for measuring adolescents' anger. Data were analyzed by using mean, standard deviation and two-way analysis of variance (ANOVA). The findings of the present study show that gender and socio-economic status have significant effect on adolescents' anger. Male respondents express more anger than female respondents. With low SES expressed more anger than the middle and high SES. Also respondents with middle SES expressed more anger than the high SES. Result also shows that there is no significant interaction between gender and socio-economic status.
\end{abstract}

Keywords: Adolescent Anger, socio-economic status, gender

\section{Introduction}

Adolescence is the period of life from late childhood until physical growth is relatively complete and also marks the beginning of early adulthood. Age 13 through 16 is considered early adolescence, and 17 through 21 is late adolescence (Wolman, 1989). The term adolescence is commonly used to describe the transitional stage of physical and psychological development between childhood and adulthood. There is no one scientific definition of adolescence or set age boundary. There are key development changes that nearly all adolescents experience during their transition from childhood to adulthood. This time frame, however, not only describes a very diverse reality, but adolescence varies considerably across cultures, over time, and within individuals (Kaplan, 2004).

Chronological age provides only a rough marker of adolescence, and scholars have found it difficult to agree upon a precise definition of adolescence (Christie et al. 2005). Adolescence is uniquely experienced within individuals even of similar chronological age. The physical onset of puberty is not the same for all adolescents. According to Kaplan (2004), Adolescence may begin as early as age 9 for some girls, or as late as age 13 for others. Thus the physical experiences of these individuals will vary greatly, as will their social experiences. Girls who mature earlier than their peers often begin "adolescence" looking older than their chronological age. Likewise, adolescence has not always lasted until age 18 or 21. For example, prior to the industrial revolution, the family functioned as an inclusive unit; thus, the transition from childhood to adulthood was relatively short. As children performed adult tasks, they became adults as early as age 13 (Sisson et al. 1987).

Asian cultures are collectivistic in nature, in which group harmony and loyal family membership are prized above individuality, autonomy or individual satisfaction (Pipher, 1994). These cultures value tradition, conformity, obedience, and fitting into family and society as opposed to the independence, individualism, exploration, and self-expression common in western cultures (Kaplan, 2004). Therefore, the lack of congruency across and within different cultures makes the term adolescence fairly inaccurate as a definition for one distinctly inclusive time period. A thorough understanding of adolescence in society depends on information from various perspectives, most importantly from the areas of psychology, biology, history, sociology, education, and anthropology. Within all of these perspectives, adolescence is viewed as a transitional period between childhood and adulthood whose cultural purpose is the preparation of children for adult roles (Larson and Wilson, 2004).

Anger is an emotional reaction characterized by extreme displeasure, rage, indignation, or hostility. Deffenbacher et al. (1996) have been defined anger as an experiential state consisting of emotional, cognitive and physiological components that co-occur, rapidly interacting with and influencing each other in such a way that they tend to be experienced as a single phenomenon. It is also described as a negative emotion in terms of subjective experience. Anger is considered to be the drive or motive behind aggressive behavior and the subjective experience that accompanies aggressive impulses (Averill, 1983). Like other emotions, anger is 
accompanied by physiological and biological changes. When a person becomes angry, his heart rate and blood pressure rises, as do levels of energy, adrenaline, noradrenalin, and hormones (Peacock, 2000).

Novaco (1985) defined anger as having four distinct components: physiological, affective, behavioral and cognitive. The affective component of anger, also referred to as anger experience, relates to the strength of emotional responses toward anger provoking situations. The behavioral component refers to coping mechanisms, which may be positive or destructive, that people use to express anger. The cognitive component reflects the types of negative beliefs or hostility, that people have about the world and in particular refers to the negative attributions they hold towards others or places. According to Izard (1977), anger is a powerful and necessary tool for survival. The innate purpose of anger is to enable the mobilization of energy so that the person becomes capable of self-defense with vigor and strength in an assertively appropriate manner. Anger potentiates a sense of personal control which enables the person to take action (Biaggio, 1987).

Anger may be conceived as existing of two types: instrumental and reactive. Instrumental anger is expressed as delayed or covert or hidden anger. This is defined as negative emotion that triggers a delayed response. This is resulting in a desired and planned goal of revenge which is internally motivated by some memory of a past provocation. Another term associated with instrumental anger is "proactive aggression" (Dodge, 1991). Researchers have suggested that instrumentally angered adolescents have histories of delinquency and antisocial behaviors and use aggression as an attempt to achieve some desired end (Goldstein et al. 1987). A reactive anger is expressed an overt anger. This type of anger is defined as an immediate angry response to a perceived threatening or fear provoking event. It is an immediate response to some angerprovoking event that is perceived as negative, threatening, or fear provoking (Asarnow and Cullen, 1985). Adolescents experiencing reactive anger are deficient when cognitively processing environmental cues. They have negative attributions, experience difficulty in solving problems, are easily physically aroused during conflict, and become hyperactive and impulsive. Lochman et al. (1989) suggested that adolescents who evidence difficulty in controlling their anger tend to have skill deficits in their cognitive processing, behavior functioning, self-control, and ability to cope in social arenas. Overall, reactive and instrumental anger differ vastly in their response timing, intensity, and frequency of occurrence. However, individuals who routinely experience either type of anger have need for specific assessment and treatment.

Socio-economic status (SES) is an economic and sociological combined total measure of an individual or family's economic and social position in relation to others, based on income, education and occupation. Socio-economic status is typically broken into three categories, high SES, middle SES, and low. Socioeconomic class means group of people within a society who possess the same socio-economic status. Belonging to a social class is not merely an objective fact, but is generally accompanied by a perception of class identity. In this sense, social class is not merely a personal attribute, but also a contextual variable that characterizes a group of people. The shared culture of a particular class influences, and is influenced by, people's attitudes and lifestyle (Krieger et al. 1997). Education also plays a role in income. Median earnings increase with each level of education. Higher levels of education are associated with better economic and psychological outcomes (i.e.: more income, more control, and greater social support and networking). Education plays a major role in skill sets for acquiring jobs, as well as specific qualities that stratify people with higher SES from lower SES.

Rahman and Huq (2005) studied to explore aggression in adolescent boys and girls as related to socioeconomic status (SES) and residential background in Bangladesh. They investigated 240 respondents and found that respondents with high SES expressed more aggression than the middle and low SES. Respondents with middle SES expressed more aggression than the low SES and girls expressed more aggression than the boys.

Taylor (2006) found that Lower and middle class respondents report feeling more anger than their upper class counterparts. Gallo and Matthews (2003) studied socio-economic class-based patterns in emotion and found lower-class individuals experience more negative emotions, such as anxiety, sadness, and anger, than upper class individuals.

Studies involving adolescents indicated no differences between boys and girls on the experience of anger (Fabes and Eisenberg, 1992; Cole et al.1994). However, others have reported significant gender differences in the expression of anger (Maccoby and Jacklin, 1980; Crick, 1997). In both these studies boys were more likely than girls to use physical expression of their anger.

Swaffer and Epps (1999), in their study of male and female adolescents, found no significant differences in either the experience or expression of anger. However, in a study of elementary, middle, and high school students, Cox et al. (2000) found boys were significantly more likely to express their anger outwardly than girls.

Boman (2003) studied gender differences in the affective, behavioral, and cognitive components of anger in 102 students. He found that girls and boys do not differ in their experience (affective) of anger and girls are more likely to express positively (behavioral) their anger than boys. Additionally, his results supported the expectation that boys are more hostile (cognitive) towards school than girls. 
Lochman et al. (1989) investigated adolescence anger and found that adolescents who evidence difficulty in controlling their anger tend to have skill deficits in their cognitive processing, behavior functioning, self-control and ability to cope in social arenas.

Adolescence is an important part for human life. As adolescence is a transitional stage of development between childhood and adulthood, girls and boys face many physiological and psychological changes in their life. The inability to cope with the changes may lead to other negative consequences such as poor academic performance, low self-esteem and low well-being. These consequences, in turn, can lead to various behavioral problems including anxiety, depression, anger, school drop-out, drug and alcohol abuse etc. Several factors have been to explain the causes of anger of adolescents. Among them Socio-economic differences and gender are employed to give a greater understanding about the explanation of anger. Most of the studies in this area have been done in western culture, but in Bangladesh there are very few studies. Moreover, previous studies showed contradictory results on adolescents' anger. So the present study will help to understand adolescent anger in our cultural context. This will increase our theoretical knowledge and may help those persons who are concerned with the development of child and adolescents. The present study will also help Counselor or psychologist to select the appropriate intervention program for the adolescents who typically express greater anger. From the insight of the above mentioned literature review and rationale the objectives of the present study were-

1. To investigate whether adolescents' anger varies according to socio-economic status.

2. To investigate whether adolescents' anger varies according to gender.

3. To investigate whether there is any interaction effect between socio-economic status and gender.

\subsection{Target population}

\section{Method}

The target population of the present study was adolescents of Chittagong district.

\subsection{Participants}

For the present study two-stages sampling procedure was used.

\subsubsection{Educational institutions and areas selection}

Educational institutions and areas were taken purposively from Chittagong district in Bangladesh. The names of the educational institutions are Chittagong University Laboratory School and College, Fatehpur Multilateral High School, Fathabad Collage and Shahjalal High School. The residential areas are south campus area of Chittagong University, shahid miner residential area of university, north campus residential area, railway slam of university station, kulal Para and Aman Bazar at Chittagong district.

\subsubsection{Respondents selection}

120 adolescent respondents were selected conveniently as study participants from the above mentioned schools and areas. Age of the respondents ranged from 13-19 years (mean $=15$ years, $\mathrm{SD}=1.61$ years). Among them $60(50 \%)$ adolescents were male and $60(50 \%)$ were female. The respondents came from different socioeconomic status. 40 (33.3\%) adolescents were from higher class, 40 (33.3\%) adolescents were from middle class and the rest of $40(33.3 \%)$ were from lower class. There education level ranged from class 6 to higher secondary. All of the respondents were physically and mentally well. The sample distribution of the present study is given below-

TABLE 1: Number of study sample according to socio-economic status and gender

\begin{tabular}{ccccc}
\hline \multicolumn{5}{c}{ Socio-economic status } \\
\hline Gender & Higher class & Middle class & Lower class & Total \\
\hline Male & 20 & 20 & 20 & 60 \\
Female & 20 & 20 & 20 & 60 \\
Total & 40 & 40 & 40 & 120 \\
\hline
\end{tabular}

\subsection{Measuring Instruments}

Two measuring instruments were used. These ares-

\subsubsection{Demographic and Personal Information Questionnaire}

A demographic and personal information questionnaire were used to collect data about participant's age, gender, education, father's occupation, father's education, father's income, mother's occupation, mother's education, mother's income, family member, total family income, socio-economic status. 


\subsubsection{Bangla version of Adolescent Anger Rating Scale (AARS)}

An adapted Bangla version of "Adolescent Anger Rating Scale" (AARS) (Islam and Chowdhury, 2011) originally developed by Burney (2001) used for measuring adolescents' anger. It is a self report Likerttype rating scale designed to measure specific expressions of anger of adolescents, ages 11 to 19 years. The questionnaire contains forty-one questions where twenty are instrumental anger based, eight are reactive anger based and thirteen are anger control based. The scale scores range from 41 to 164. Higher anger scores reflect greater endorsements of anger. Twenty-eight questions are positive and thirteen are negative. There are four alternatives for each question. The alternative answers for showing anger in positive items are, a. 1 for Hardly Ever, b. 2 for Sometimes, c. 3 for Often and d. 4 for Very often. Moreover, the scoring system for negative item is just opposite of the scoring system of positive item i.e. a. 4 for Hardly Ever, b. 3 for Sometimes, c. 2 for Often and d. 1 for Very often. Reliabilities of the original version ranged from 0.81 to 0.96 . The convergent and discriminant validity of the original version are 0.57 and 0.46 respectively. The test-retest, spilt-half (odd-even) and Cronbach Alpha reliability of the AARS Bangla version are 0.90, 0.75 and 0.78 respectively. The Bangla version also assured high level of content and construct validity.

\subsection{Procedure}

For collecting relevant data from the participants, at first permission from the school authorities and guardians were taken. Respondents were told that the sole purpose of the investigation was academic and their response would be kept confidential. Before administration of the questionnaire, necessary rapport was established with respondents. Then the Adolescent Anger Rating Scale was administered to respondents and requested a silent reading at the instruction provided with the scale before starting to answer. They were also requested not to omit any item in the scale and they were encouraged to answer all the items by telling that, there is no right or wrong answer to any item. All possible clarifications were made to the problems if faced by the respondents. There was no time limit for the respondents to answer all the items of the scale. After completing of their tasks the answered questionnaires were collected from them and they were given thanks for their sincere co-operation.

\section{Results and discussion}

The data were analyzed by using mean, standard deviation and two-way analysis of variance (ANOVA). All statistical analyses were carried out using the statistical program SPSS version 16.0 for windows.

TABLE 2: Descriptive statistics of adolescents' anger according to gender and socio-economic status

\begin{tabular}{lllll}
\hline & \multicolumn{4}{c}{ Socio-economic status } \\
\hline Gender & Higher class & Middle class & Lower class & Total \\
\hline Male & $M=75.85$ & $M=73.05$ & $M=88.25$ & $M=79.05$ \\
& $S D=9.91$ & $S D=13.72$ & $S D=15.75$ & $S D=14.71$ \\
Female & $M=61.40$ & $M=74.05$ & $M=78.35$ & $M=71.26$ \\
& $S D=9.71$ & $S D=10.29$ & $S D=15.10$ & $S D=13.77$ \\
Total & $M=68.62$ & $M=73.55$ & $M=83.30$ & \\
& $S D=12.14$ & $S D=11.98$ & $S D=15.98$ & \\
\hline
\end{tabular}

Table 1 shows that adolescents' anger score differ according to male $(M=79.05, S D=14.71)$ and female $(M=71.26, S D=13.77)$ and according to socio-economic status, higher class $(M=68.62, S D=12.14)$, middle class $(M=73.55, S D=11.98)$, lower class $(M=83.30, S D=15.98)$. To investigate the main and interaction effect of socio-economic status and gender on adolescents' anger score, two-way analysis of variance (ANOVA) was computed.

TABLE 3: Summery of the analysis of variance of adolescents' anger score according to gender and socio-economic status

\begin{tabular}{cccccc}
\hline Source of variance & $d f$ & $S S$ & $M S$ & $F$ & Sig.(p-value) \\
\hline Gender & 1 & 1817.408 & 1817.408 & 11.354 & .001 \\
socio-economic status & 2 & 4462.317 & 2231.158 & 13.939 & .022 \\
Gender * socio economic status & 2 & 1260.717 & 630.358 & 3.938 & .380 \\
Error & 114 & 18247.550 & 160.066 & & \\
Corrected total & 119 & 25787.992 & & & \\
\hline
\end{tabular}

Table 3 shows that gender $(F=11.354, d f=1,119, \mathrm{p}<.01)$ and socio-economic status $(F=13.393, d f=2$, $119, \mathrm{p}<.05)$ have significant effect on adolescents' anger. It indicates that adolescents' anger vary as a function 
of gender and socio-economic status. The result also shows no significant interaction effect between gender and socio-economic status.

Adolescents with low SES expressed more anger $(M=83.30, S D=15.98)$ than the middle and high SES. Also respondents with middle SES expressed more anger $(M=73.55, S D=11.98)$ than the high SES $(M=$ $68.62, S D=12.14)$. This finding is supported by Taylor (2006). SES affects human functioning in many ways, including development across the life span, psychological and physical health. Low SES and its correlates, such as lower education, poverty, and poor health, ultimately affect our society as a whole; in particular, Bangladeshi children face one of the highest rates of poverty within the industrialized world. Lower socio-economic class is associated with coping with more threatening environments than their upper-class counterparts. Low income families focus on meeting immediate needs and do not accumulate wealth that could be passed on to future generations. This outward orientation can lead to increased more emotional and anger experiences. Family income also has a profound influence on the educational opportunities available to adolescents and on their chances of educational success. Due to residential stratification and segregation, low-income students usually attend schools with lower funding levels, which result in reduced availability of textbooks and other instructional materials. The effects of concentrated poverty in schools may include disciplinary problems and chaotic learning environments. These school characteristics, combined with limited parental involvement in adolescents' conceptualization of socially accepted norms and values, have serious consequences like aggression and other deviant behaviors. Higher class parents can take an active role in their children's education and development by using controlled organized activities and fostering a sense of entitlement through encouraged discussion. Families with lower income do not participate in this movement.

The mean scores indicate that adolescents' anger score of male $(M=79.05, S D=14.71)$ was higher than score of female $(M=71.26, S D=13.77)$. It was partially supported by Cox et al. $(2000)$. In general, much research has suggested that males use more physical aggression than females and females use more verbal aggression than males. Female adolescents seem to suppress anger expression more than males. Regarding anger expression, females are more likely to have positive coping mechanisms. These coping mechanisms are all of a passive nature. For example, girls are more likely to share their feelings or talk things over with someone else when angry. But males are more likely to show anger by indicating destructive coping. For example, boys are more likely to break things or disrupt a class when angry. According to theories of aggression by Parke \& Slaby (1983), gender role and learning play a key role for anger and aggressive behavior of males. Males are exposed to parenting practices that promote rough-and-tumble, anger and aggressive behaviors whereas females are exposed to parenting practices that promote caring and close interpersonal relationships. These differential socialization practices appear to foster adolescents' anger to a greater extent in males than in females.

The nature of changes and challenges that occur simultaneously during adolescence require the development of effective coping strategies for adaptive functioning. During this period one may experiences peer rejection, academic failure, and lowered self-esteem. He or she continues to be persistently aggressive in interactions with both peers and adults and is at risk for social rejection. Arguments with parents and siblings may increase and the adolescent may develops other significant behavior problems. School difficulties such as truancy, increased detentions and academic detainment, substance abuse and adolescent health problems may all be linked to aggressive and antisocial behavior patterns. To be healthy and safe, adolescents need to be equipped with skills that allow them to cope with anger in a productive manner.

\section{Conclusion}

In today's society, adolescents face daily situations that contribute to them being angry. A multitude of factors contribute to their anger. Some of these factors include poverty, physical or psychological abuse, neglect, alcoholism etc. A major contribution of the current study is the exploration of the association of adolescent's anger with socio-economic status of Chittagong district in Bangladesh. But the sample size was relatively small and the study was administered to some specific areas of Chittagong. Future research can be carried out on a large scale sample covering different areas of Bangladesh. Further studies are needed to measure and investigate the components (instrumental anger, reactive anger and anger control) of anger. Future studies may also wish to explore the passive/active aspects of anger coping and its relationship to socio-economic status, gender and other demographic factors. In the school setting, students don't receive anger management counseling. Intervention programs and counseling designed to help adolescents to cope in healthy ways with their anger could be advised to address differentially the needs of angry females and males. It may be very helpful for teachers to pay attention to statements made by students that suggest an underlying hostility towards school. This could be useful in preventing some later behavioral problems, both in the classroom and schoolyard, which reflect anger coping strategies. Parental support and monitoring also can help as protective factors against adolescents' anger. The combination of support and monitoring that characterizes authoritative parents can contribute to lower involvement in aggressive and problem behaviors of adolescents. 


\section{References}

[1]. Asarnow, J. R. and Cullen, J. W. (1985). Boys with peer adjustment problems: Social cognitive processes. Journal of Consulting Clinical Psychology, 53, 80-87.

[2]. Averill, J. R. (1983). Studies on anger and aggression: Implications for theories of emotion. American Psychologist, 38, 1145-1159.

[3]. Biaggio, M.K. (1987). Clinical dimensions of anger management. American Journal of Psychotherapy, 41(3), $417-427$.

[4]. Boman, P. (2003). Gender Differences in School Anger. International Education Journal, 4(2), 71-77.

[5]. Burney, D. M. (2001). Initial development and score validation of the Adolescent Anger Rating Scale. Educational and Psychological Measurement, 61, $446-460$.

[6]. Christic, D. and Viner, R. (2005). Adolescent development. British Medical Journal, 330, 301-400.

[7]. Cole, Z.W.C., Richardson, P. M. and Friedman, R. J. (1994). Social problem solving in disruptive preschool children: Reactions to hypothetical situations of conflict and distress. Merrill-Palmer-Quarterly, 40, 98-119.

[8]. Cox, D.L., Stabb, S.D. and Hulgus, J.F. (2000). Anger and depression in girls and boys: A study of gender differences. Psychology of Women Quarterly, 24, 110-112.

[9]. Crick, N. R. (1997). Engagement in gender normative versus no normative forms of aggression: Links to social-psychological adjustment. Developmental Psychology, 33 (4), 610-617.

[10]. Deffenbacher, J. L., Lynch, R. S., Oetting, E. R. and Kemper, C. C. (1996). Anger reduction in early adolescents. Journal of Counseling Psychology, 43, 149-157.

[11]. Dodge, K. A. (1991). The structure and function of reactive and proactive aggression. In D. J. Pepler and K. H. Rubin (Eds.), the development and treatment of childhood aggression, 201-218.

[12]. Fabes, R.A. and Eisenberg, N. (1992). Young children's coping with interpersonal anger. Child Development, 63, 116-128.

[13]. Gallo, L. C. and Matthews, K. A. (2003). Understanding the association between socioeconomic status and physical health: Do negative emotions play a role? Psychological Bulletin, 29, 10-51.

[14]. Goldstein, A. P., Glick, B., Zimmerman, D. and Reiner, S. (1987). Aggression replacement training: A comprehensive intervention for the acting-out delinquent. Champaign, IL: Research Press.

[15]. Islam, S. and Chowdhury, L. (2011). Bangla Adolescent Anger Rating Scale (AARS) for use in Bangladesh. The Dhaka University Journal of Psychology, 35, 21-30.

[16]. Izard, C. (1977). Human emotions, New York, NY: Plenum Press.

[17]. Kaplan, P. S. (2004). Adolescence. Boston: Houghton Mifflin Company, 1, 4, 81

[18]. Krieger, N., Williams, D. R. and Moss, N. E. (1997). "Measuring Social Class in U.S. Public Health; Research: Concepts, Methodologies, and Guidelines." Annual Review of Public Health, 18, 341-378.

[19]. Larson, R. and willson, S. (2004). Adolescence across place and time: Globalization and the Changing pathways to adulthood. In Lerner, R. and Steinberg, L. Handbook of adolescent psychology. New York: Wiley.

[20]. Lochman, J. E., Lampron, L. B. and Rabiner, D. L. (1989). Format and salience effects in the social problem-solving of aggressive and nonaggressive boys. Journal of Clinical Child Psychology, 18, 230-236.

[21]. Maccoby, E. E. and Jacklin, C. N. (1980). Sex differences in aggression: A rejoinder and reprise. Child Development, 51, 964-980.

[22]. Novaco, R. W. (1985). Anger as a clinical and social problem. Advances in the study of aggression, 1. New York: Academic Press.

[23]. Parke, R.D. and Slaby, R.G.(1983). The development of aggression. In P.H. Mussen (Series Ed.) and Hetherington, E.M. (Vol. Ed.), Handbook of child psychology, Vol.4, Socialization, personality, and social development (4th Ed.). New York: Wiley.

[24]. Peacock, J. (2000) Anger management. Gaining control of your anger. Mankato, MN: Capstone Press.

[25]. Pipher, M. (1994). Reviving Ophelia. New York: Ballantine Books. Averill, J.R.(1983). Studies on anger and aggression. American Psychologist, 38, 1145-1159.

[26]. Rahman, A. K. M. R. and Huq M. M. (2005). Aggression in adolescent boys and girls as related to socio-economic status and residential background. Journal of life Earth Science, 1(1), 5-9.

[27]. Sisson, L. A., Herson, M. and Van, H. V. B. (1987). Historical perspectives. In Handbook of Adolescent Psychology, Oxford: Pergamon Press, 1-7.

[28]. Swaffer, T. and Epps, K. (1999). The psychometric assessment of anger in male and female adolescents resident at a secure you th treatment centre. Journal of Adolescence, 22, 419-422.

[29]. Taylor, T. (2006). Lower, Working and middle class. Chicago: Barbara Press.

[30]. Wolman, B. B. (1989). Dictionary of behavioral science. San Diego, CA: Academic Press. 